\title{
P24 antigen detection, viral isolation, DNA-PCR and in vitro antibody production for the diagnosis of HIV-1 latent infection in heterosexual women at high risk for HIV-1 infection
}

\author{
M Di Stefano, J R Fiore, M Chironna, G Buccoliero, C Romanelli, L La Grasta, \\ $M$ Quarto, G Angarano, G Pastore
}

\begin{abstract}
Introduction-The report of the existence of at-risk seronegative subjects, latently infected with HIV-1 and producing "in vitro" HIV-1 specific antibodies, prompted the authors to evaluate extensively twenty-five heterosexual HIV-1 seronegative women at high risk for HIV-1 infection.

Material and methods-The capability of peripheral blood mononuclear cells from such subjects to produce "in vitro" HIV-1 specific antibodies after pokeweedmitogen stimulation, was studied. Silent HIV-1 infection was investigated by HIV-1 DNA PCR, viral isolation and serum p24 Ag detection at entry and after 6 and 12 months.
\end{abstract}

Results-Three seroconversions took place within 12 months, but no HIV-1 infections were found in the absence of detectable serum anti HIV-1 antibodies, even in subjects who apparently produced such antibodies in vitro. The antibodies produced in vitro by the seronegative women studied appeared of narrow specificity, reacting mainly with gp 160/120 envelope glycoproteins.

Conclusions-A strong concordance was found between the serological status and the other markers for HIV-1 infection, suggesting that the phenomenon of HIV-1 "latent infection" is a very rare event, if it occurs at all. Seronegative women sexually exposed to the virus may produce in vitro anti HIV-1 antibodies of narrow specificity in the absence of other signs of infection and this phenomenon might be related to an anamnestic response to the virus.

(Genitourin Med 1995;71:123-125)

Diseases, University of

Bari, Bari, Italy

M Di Stefano

J R Fiore

G Buccoliero

C Romanelli

L La Grasta

G Angarano

G Pastore

Institute of Hygiene

M Chironna

M Quarto

Correspondence to:

Professor G Pastore

Clinic of Infectious Diseases,

University of Bari,

Policlinico Hospital,

Piazza Giulio Cesare

70124-Bari, Italy.

Accepted for publication

19 December 1994 found any evidence of latent infection in "at high risk" seronegative subjects. ${ }^{7-11}$

Recent reports also indicate that peripheral blood mononuclear cells (PBMC) from cer- tain HIV-1 seronegative subjects at high risk for HIV-1 infection, may be induced "in vitro" to produce HIV-1 specific antibodies. ${ }^{12} 13$ The specificity of such antibodies in Western Blot analyses was mainly against the gp $160 / 120$ envelope glycoproteins. In one of these studies, ${ }^{12} 12$ of 26 seronegative antibody producers were found to carry HIV-1 as detected by PCR. This observation strengthens the hypothesis that subjects harbouring HIV-1 do exist in spite of a lack of detectable serum antibodies, and also suggests that "in vitro antibody production" (IVAP) might be a useful tool with which to detect HIV-1 latently infected individuals.

An important limitation of these studies has been the lack of clinical and serological follow up of the patients. It is therefore unknown whether these seronegative antibody producers undergo overt seroconversion over time. The clinical relevance of the phenomenon of "in vitro" antibody production is thus still undefined.

In this study $25 \mathrm{HIV}-1$ seronegative women at very high risk for HIV-1 infection were tested for HIV-1 IVAP, evaluated for the presence of HIV-1 latent infection using DNA-PCR, viral isolation and serum p24 Ag detection and followed over time to detect seroconversions.

\section{Material and methods \\ Patients}

Twenty-five women, who were the regular sexual partners of HIV-1 seropositive individuals selected from participants in an ongoing cohort study on HIV-1 heterosexual transmission at the Clinic of Infectious Diseases, University of Bari, were studied.

Regular sexual partners were defined as HIV-1 seronegative subjects who had had a stable and monogamous heterosexual relationship (in which they had had unprotected sex for at least 6 months before blood sampling) with an individual known to have antibodies to HIV-1 and who had not been exposed to any other known risk factor for HIV-1 infection. The women were seen and tested for anti HIV-1 antibodies at entry in the study and after 6 and 12 months. The control group consisted of five women known to be positive for anti HIV-1 antibodies.

\section{Serological tests}

HIV-1 specific antibodies were tested, at entry and after 6 and 12 months by a commercial 
Table 1 Virological and serological evaluation of at risk heterosexual women seronegative and seropositive for $H I V-1$

\begin{tabular}{lcclllll}
\hline & No & Months $^{*}$ & $A b$ & IVAP & $p 24$ & HIV isol & $P C R$ \\
\hline Seronegative & 25 & 0 & 0 & 5 & 0 & 0 & 0 \\
women & & 6 & 1 & nd & 0 & 1 & 1 \\
Seropositive & 5 & 12 & 3 & nd & 1 & 3 & 3 \\
women & & & 5 & 5 & 3 & 5 & 5
\end{tabular}

^Anti HIV-1 antibodies (Ab), in vitro antibody production (IVAP), serum p24 Ag (p24), HIV-1 isolation in cell culture (HIV isol) and HIV-1 DNA PCR during 12 months of follow-up

ELISA (Behring Werke, Marburg, Germany) and Western Blot analyses (Biotech Research Laboratories, Inc., Rockville, Maryland, U.S.A.). The presence of serum p24 Ag was tested on the same occasions using a commercially available antigen-capture assay (Du Pont, De Nemours, Wilmington, Delaware, USA).

\section{Virus detection}

Viral isolation and DNA PCR analyses were performed for each woman at entry to the study and after 6 and 12 months of follow-up. Virus was isolated from peripheral blood mononuclear cells (PBMC) by a co-cultivation technique described elsewhere. ${ }^{14}$ For PCR analyses, PBMC samples were tested for the presence of HIV-1 DNA with two sets of nested primers specific for regions of the POL and ENV genes (JA17 to JA20 and JA13 to JA16, respectively)..$^{15}$

The PCR products were analysed by electrophoresis on $1.5 \%$ agarose gel stained with ethidium bromide. Negative and positive controls were included in each run.

\section{IVAP test}

The IVAP test was performed, at entry, by pokeweed-mitogen (PWM) stimulation of peripheral blood mononuclear cells as described elsewhere. ${ }^{13}$ The presence of HIV-1 specific antibodies in the supernatants was tested by ELISA and Western Blot. ${ }^{13}$

\section{Results}

The results are summarised in table 1 . In five seronegative subjects it was possible to demonstrate production in vitro of HIV-1 specific antibodies after stimulation of PBMC with PWM. The specificity of antibodies detected in these IVAP supernatants was mainly against gp 160/120 envelope glycopro-

Table 2 Specificity of anti HIV-1 antibodies detected in the IVAP assay in women seronegative and seropositive for HIV -1

\footnotetext{
Seronegative women:

Patient 3. $160 / 120$

Patient 3: gp 160/120

Patient 9: gp 160/120

Patient 14: gp 160

Patient 21: gp 160/120

Seropositive women:

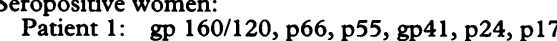

$\begin{array}{ll}\text { Patient 1: } & \text { gp 160/120, p66, p55, gp41, } \\ \text { Patient 2: } & \text { gp 160/120, p66, gp41, p24 }\end{array}$

$\begin{array}{ll}\text { Patient 2: } & \text { gp 160/120, p66, gp41, p24 } \\ \text { Patient 3: } & \text { gp 160/120, p55, p24, p17 }\end{array}$

Patient 4: gp 160/120, p66, p55, p51, gp41, p31, p24, p17

Patient 5: gp 160/120, gp41, p24, p17 ^Antibody reactivity of cultural supernatants from IVAP
assays, as detected in Western Blot.
}

teins, while PWM-stimulated PBMC from seropositive women did produce in vitro antibodies reacting with several HIV-1 structural proteins (table 2 ).

As shown in table 1, no signs of HIV-1 latent infection were documented in the study group. Serum p24 Ag and cell cultures for viral isolation were negative and no HIV-1 specific DNA sequences could be amplified by DNA-PCR in individuals negative for HIV-1 specific antibodies. Three women seroconverted during follow-up, after 6 (1 case) and 12 months (2 cases). All the seroconversions were found in the IVAP negative group. No evidences of latent HIV-1 infection could be found prior to these 3 seroconversions.

The seropositive individuals, both controls and the seroconverting subjects, were also positive for viral isolation and DNA-PCR, and in four cases for serum p24 Ag.

\section{Discussion}

We studied 25 HIV-1 seronegative women who were steady sexual partners of infected men; five of these women were positive in the IVAP test (IVAP + ) since their PBMC were capable, after PWM stimulation, of producing in vitro HIV-1 specific antibodies, mainly directed against gp 160/120 envelope glycoproteins. Such a phenomenon could be observed only in subjects "at high risk" for HIV-1 infection. In fact, in a previous study ${ }^{13}$ we were not able to induce in vitro production of HIV-1 specific antibodies in a control group of 10 (5 men and 5 women) healthy blood donors with no known risk factor for HIV-1 infection.

In contrast, the antibodies produced in vitro by HIV-1 seropositive women were of broad specificity, and reacted with several HIV-1 structural proteins on the Western Blot test.

In spite of repeated sexual exposure to the virus in our study population, we did not detect any sign of HIV-1 latent infection, either in the IVAP - or in the IVAP + group. In fact, serum p24 Ag, viral cultures and DNA-PCR analyses were negative in all cases. This finding is in disagreement with the results of Jehuda Cohen et $a l^{12}$ who found HIV-1 DNA sequences by PCR in roughly $50 \%$ of a group of IVAP positive subjects.

We do not have explanations for these discrepancies. Our PCR protocol appeared to be highly sensitive and able to detect as few as 10 proviral copies in the sample. Thus we do not believe that the cause could be a poor sensitivity of our PCR technique. Two explanations have to be considered. Either the possible different epidemiological settings in the two studies might at least in part, be responsible for such differences; or the PCR protocol in the study of Jehuda Cohen involved a DNA extraction step that might have caused laboratory contamination. Our PCR protocol does not need such a procedure. False positive results must be taken into account, especially as the latent infections reported in the study 
of Jehuda Cohen were not confirmed by other tests (viral isolation, p24 Ag) or in subsequent samples.

During the follow-up it was possible to document three seroconversions (after 6 and 12 months); interestingly all of these occurred in the IVAP negative group. None of the IVAP + subjects underwent an overt seroconversion. This indicates that the phenomenon of the in vitro production of HIV-1 specific antibodies in HIV-1 seronegative individuals is not related to a condition of latent infection and is not predictive of subsequent overt seroconversion.

A further finding from our study is the strong concordance between the serological status of the subjects and the other markers for HIV-1 infection that were evaluated (DNA-PCR, viral isolation, p24 Ag detection). Indeed, no latent infections were detected in HIV-1 seronegative subjects, even in those who seroconverted a few months later. It is generally thought that the interval between infection and seroconversion is less than three months and our findings support this, indicating that the phenomenon of HIV-1 latent infection in "at high risk" seronegative subjects seems to be a very rare event, if it occurs at all.

A recent report ${ }^{16}$ demonstrated that recently exposed homosexual men may show an anamnestic response to HIV-1 in the T cell compartment, in the absence of both HIV-1 specific antibody production and latent infection (as detected by viral isolation and PCR). Our results might be considered in line with the observations from this latter study. It is conceivable that women in our study have been locally (sexually) exposed to low levels of HIV-1 antigens or to inactivated virus, with no, or only transient antibody response; in this case the B-cell responsiveness could be detected only after mitogen stimulation. This might be supported by the finding from this study of the narrow specificity of the antibodies produced in vitro by seronegative subjects (mainly against gp 160/120 envelope glycoproteins) and by the observation (Fiore J.R., unpublished data) of HIV-1 specific anti- bodies, directed against gp $160 / 120$ in the vaginal washing from certain at high risk seronegative women.

This work was supported in part by grants from Instituto Superiore di Sanita (Projetto AIDS, Grant 9203-20).

We are grateful to A. La Gioia and D. Gatti for their invaluable technical assistance. Mariantonietta Di Stefano is supported by a fellowship of the Istituto Superiore di Sanitá.

1 Ranki A, Valle S, Krohn M, et al. Long latency precedes overt seroconversion in sexually transmitted human immunodeficiency virus infection. Lancet 1987;ii: 589-93.

2 Farzadegan $\mathrm{H}$, Polis $\mathrm{M}$, Wolinsky $\mathrm{S}$, et al. Loss of human immunodeficiency virus type-1 antibodies with evidence of viral infection in asymp

3 Ou C, Kwok S, Mitchell S, et al. DNA amplification for direct detection of HIV-1 in DNA from peripheral blood direct detection of HIV-1 in DNA from periphe
mononuclear cells. Science 1988;239:295-7.

4 Imagawa D, Lee $M$, Wolinsky S. Human immunodeficiency virus type-1 infection in homosexual men who remain seronegative for prolonged periods. $N \mathrm{Engl} \mathcal{F} \mathrm{Med}$ 1989;320:1458-62.

5 Aiuti F, Ensoli F, Fiorelli V. Human immunodeficiency virus type-1 in homosexual men who remain seronegative for prolonged periods. N Engl ₹ Med 1989;321:1679.

6 Wolinsky S, Rinaldo C, Kwok S, et al. Human immunodeficiency virus type-1 (HIV-1) infection a median of 18 months before a diagnostic Western Blot. Ann Int Med 1989;111:961-72.

7 Horsburgh C, Ou C, Jason J, et al. Duration of human immunodeficiency virus infection before detection of immunodeficiency virus infection.

8 Gibbons J, Cory J, Hewlett I. Silent infections with human immunodeficiency virus type 1 are highly unlikely in multitransfused seronegative hemophiliacs. Blood 1990;76:1924-6.

9 Lifson A, Stanley M, Pane J, et al. Detection of human immunodeficiency virus DNA using the polymerase chain reaction in a well characterized group of homosexual and bisexual men. F Infect Dis 1990;161:436-9.

10 Mariotti M, Lefrere J, Noel B, et al. DNA amplification of HIV-1 in seropositive individuals and in seronegative at risk individuals. AIDS 1990;4:633-7.

11 Crowe S, Elbeik T, Ulrich P, et al. Lack of evidence of occult human immunodeficiency virus in seronegative 1991;35:160-4.

12 Jehuda Cohen T, Slade B, Powell J, et al. Polyclonal B cell activation reveals antibodies against human immunodeficiency virus type-1 (HIV-1) in HIV-1 seronegative individuals. Proc Natl Acad Sci USA 1990;87:3972-6.

13 Fiore JR, Angarano G, Fico C, et al. Pokeweed-mitogen stimulated peripheral blood mononuclear cells from at risk seronegative subjects produce in vitro HIV-1 specific antibodies. AIDS 1991;5:1034-6.

14 Fiore JR, Calabro ML, Angarano G, et al. HIV-1 variability and progression to AIDS: a longitudinal study. $\mathcal{F} \mathrm{Med}$ Virol 1990;32:252-6.

15 Albert J, Fenyö EM. Simple, sensitive and specific detection of human immunodeficiency virus type 1 in clinical specimens by polymerase chain reaction with nested primers. $\mathcal{F}$ Clin Microbiol 1990;28:1560-4.

16 Clerici M, Giorgi J, Chou C, et al. Cell-mediated immune response to human immunodeficiency virus (HIV) type 1 in seronegative homosexual men with recent sexual exposure to HIV-1. F Infect Dis 1992;165:1012-9. 